\title{
COMPARATIVE ANALYSIS OF FILTERS AND WAVELET BASED THRESHOLDING METHODS FOR IMAGE DENOISING
}

\author{
Anutam $^{1}$ and Rajni ${ }^{2}$ \\ ${ }^{1}$ Research Scholar SBSSTC, Ferozepur, Punjab \\ anutam. bansal@gmail. com \\ ${ }^{2}$ Associate Professor SBSSTC, Ferozepur, Punjab \\ rajni_c123@yahoo.co.in
}

\begin{abstract}
Image Denoising is an important part of diverse image processing and computer vision problems. The important property of a good image denoising model is that it should completely remove noise as far as possible as well as preserve edges. One of the most powerful and perspective approaches in this area is image denoising using discrete wavelet transform (DWT). In this paper comparative analysis of filters and various wavelet based methods has been carried out. The simulation results show that wavelet based Bayes shrinkage method outperforms other methods in terms of peak signal to noise ratio (PSNR) and mean square error(MSE) and also the comparison of various wavelet families have been discussed in this paper.
\end{abstract}

\section{KEYWORDS}

Denoising, Filters, Wavelet Transform, Wavelet Thresholding

\section{INTRODUCTION}

Applications of digital world such as Digital cameras, Magnetic Resonance Imaging (MRI), Satellite Television and Geographical Information System (GIS) have increased the use of digital images. Generally, data sets collected by image sensors are contaminated by noise. Imperfect instruments, problems with data acquisition process, and interfering natural phenomena can all corrupt the data of interest. Transmission errors and compression can also introduce noise [1]. Various types of noise present in image are Gaussian noise, Salt \& Pepper noise and Speckle noise. Image denoising techniques are used to prevent these types of noises while retaining as much as possible the important signal features [2]. Spatial filters like mean and median filter are used to remove the noise from image. But the disadvantage of spatial filters is that these filters not only smooth the data to reduce noise but also blur edges in image. Therefore, Wavelet Transform is used to preserve the edges of image [3]. It is a powerful tool of signal or image processing for its multiresolution possibilities. Wavelet Transform is good at energy compaction in which small coefficients are more likely due to noise and large coefficients are due to important signal feature. These small coefficients can be thresholded without affecting the significant features of the image.

Dhinaharan Nagamalai et al. (Eds) : ACITY, WiMoN, CSIA, AIAA, DPPR, NECO, InWeS - 2014 
This paper is organized as follows: Section 2 presents Filtering techniques. Section 3 discusses about Wavelet based denoising techniques and various thresholding methods. Finally, simulated results and conclusion are presented in Section 4 and 5 respectively.

\section{FILTERING TECHNIQUES}

The filters that are used for removing noise are Mean filter and Median filter.

\subsection{Mean Filter}

This filter gives smoothness to an image by reducing the intensity variations between the adjacent pixels [4]. Mean filter is also known as averaging filter. This filter works by applying mask over each pixel in the signal and a single pixel is formed by component of each pixel which comes under the mask. Therefore, this filter is known as average filter. The main disadvantage of Mean filter is that it cannot preserve edges [5].

\subsection{Median Filter}

Median filter is a type of non linear filter. Median filtering is done by, firstly finding the median value across the window, and then replacing that entry in the window with the pixel's median value [6]. For an odd number of entries, the median is simple to define as it is just the middle value after all the entries are made in window. But, there is more than one possible median for an even number of entries. It is a robust filter. Median filters are normally used as smoothers for image processing as well as in signal processing and time series processing [5].

\section{WAVELET TRANSFORM}

In Discrete Wavelet Transform (DWT), signal energy is concentrated in a small number of coefficients .Hence, wavelet domain is preferred. DWT of noisy image consist of small number of coefficients having high SNR and large number of coefficients having low SNR. Using inverse DWT, image is reconstructed after removing the coefficients with low SNR [3]. Time and frequency localization is simultaneously provided by Wavelet transform. In addition, Wavelet methods are capable to characterize such signals more efficiently than either the original domain or transforms such as the Fourier transform [7].

The DWT is identical to a hierarchical sub band system where the sub bands are logarithmically spaced in frequency and represent octave-band decomposition. When DWT is applied to noisy image, it is divided into four sub bands as shown in Figure 1(a).These sub bands are formed by separable applications of horizontal and vertical filters. Finest scale coefficients are represented as sub bands LH1, HL1 and HH1 i.e. detail images while coarse level coefficients are represented as LL1 i.e. approximation image [8] [3]. The LL1 sub band is further decomposed and critically sampled to obtain the next coarse level of wavelet coefficients as shown in Fig. 1(b). 


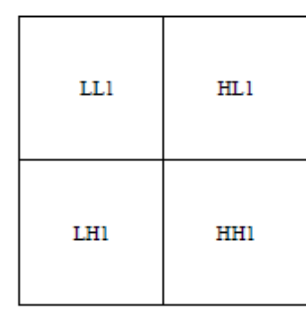

(a) One- Level

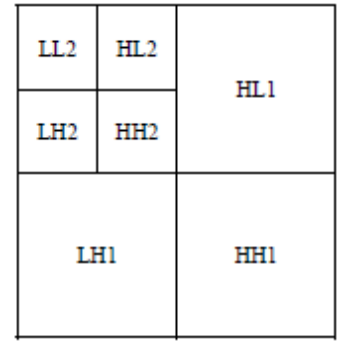

(b) Two- Level

Figure1. Image Decomposition by using DWT

LL1 is called the approximation sub band as it provides the most like original picture. It comes from low pass filtering in both directions. The other bands are called detail sub bands. The filters $\mathrm{L}$ and $\mathrm{H}$ as shown in Fig. 2 are one dimensional low pass filter (LPF) and high pass filter (HPF) for image decomposition. HL1 is called the horizontal fluctuation as it comes from low pass filtering in vertical direction and high pass filtering in horizontal direction. LH1 is called vertical fluctuation as it comes from high pass filtering in vertical direction and low pass filtering in horizontal direction. HH1 is called diagonal fluctuation as it comes from high pass filtering in both the directions. LL1 is decomposed into 4 sub bands LL2, LH2, HL2 and HH2. The process is carried until some final scale is reached. After $\mathrm{L}$ decompositions a total of $\mathrm{D}(\mathrm{L})=3 * \mathrm{~L}+1$ sub bands are obtained .The decomposed image can be reconstructed using are construction filter as shown in Figure 3. Here, the filters $\mathrm{L}$ and $\mathrm{H}$ represent low pass and high pass reconstruction filters respectively.

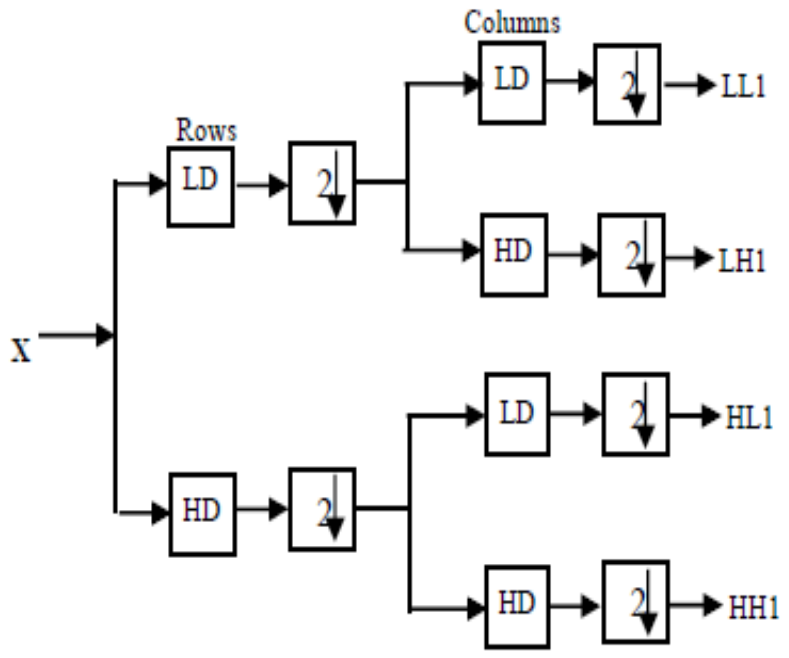

Figure2. Wavelet Filter bank for one-level Image Decomposition 


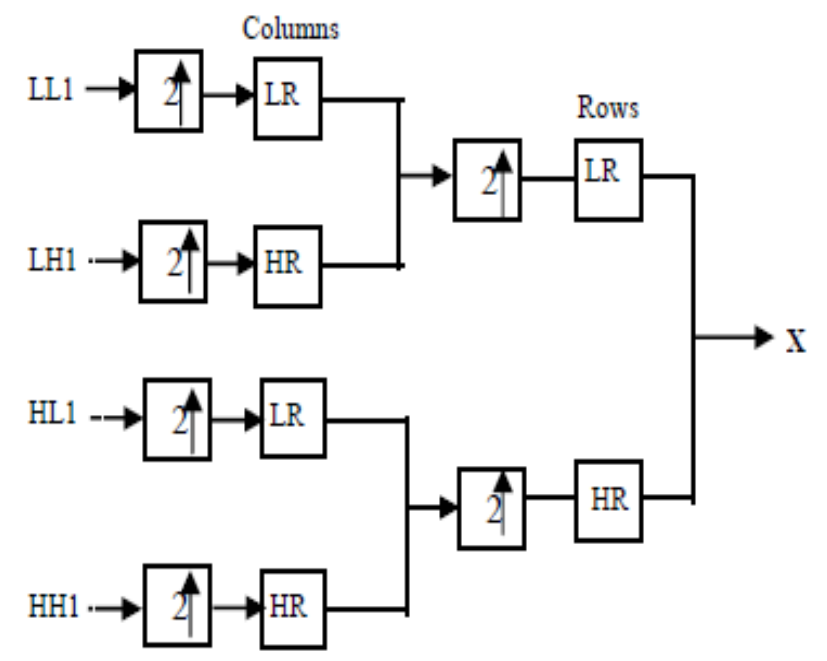

Figure3. Wavelet Filter bank for one-level Image Reconstruction

\subsection{Wavelet Based Thresholding}

Wavelet thresholding is a signal estimation technique that exploits the capabilities of Wavelet transform for signal denoising. It removes noise by killing coefficients that are irrelevant relative to some threshold [8] .Several studies are there on thresholding the Wavelet coefficients. The process, commonly called Wavelet Shrinkage, consists of following main stages:

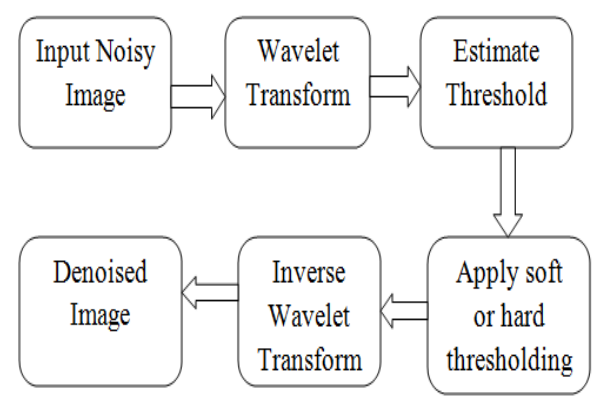

Figure 4. Block diagram of Image denoising using Wavelet Transform

- Read the noisy image as input

- Perform DWT of noisy image and obtain Wavelet coefficients

- Estimate noise variance from noisy image

- Calculate threshold value using various threshold selection rules or shrinkage rules

- Apply soft or hard thresholding function to noisy coefficients

- Perform the inverse DWT to reconstruct the denoised image.

\subsubsection{Thresholding Method}

Hard and soft thresholding is one of the thresholding techniques which are used for purpose of image denoising. Keep and kill rule which is not only instinctively appealing but also introduces artifacts in the recovered images is the basis of hard thresholding [9] whereas shrink and kill rule which shrinks the coefficients above the threshold in absolute value is the basis of soft thresholding [10]. As soft thresholding gives more visually pleasant image and reduces the 
abrupt sharp changes that occurs in hard thresholding, therefore soft thresholding is preferred over hard thresholding [11] [12].

The Hard Thresholding operator [13] is defined as,

$\mathrm{D}(\mathrm{U}, \lambda)=\mathrm{U}$ for all $\mid \mathrm{Ul}>\lambda$

$$
=0 \text { otherwise }
$$

The Soft Thresholding operation the other hand is defined as ,

$\mathrm{D}(\mathrm{U}, \lambda)=\operatorname{sgn}(\mathrm{U}) * \max (0, \mid \mathrm{UI}-\lambda)$

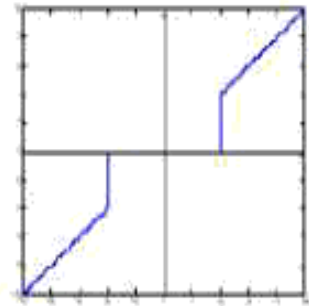

(a) Hard Thresholding

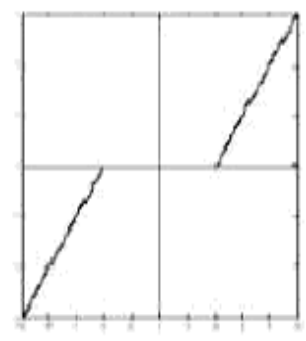

(b) Soft Thresholding [14]

Figure 5. Thresholding Methods

\subsubsection{Threshold Selection Rules}

In image denoising applications, PSNR needs to be maximized, hence optimal value should be selected [8]. Finding an optimal value for thresholding is not an easy task. If we select a smaller threshold then it will pass all the noisy coefficients and hence resultant images may still be noisy but larger threshold makes more number of coefficients to zero, which provides smoothness in image and image processing may cause blur and artifacts, and hence the resultant images may lose some signal values [15].

\subsubsection{Universal Threshold}

$$
T=\sigma \sqrt{2 \log M}
$$

where $\sigma^{2}$ being the noise variance and M is the number of pixels [16] .It is optimal threshold in asymptotic sense and minimizes the cost function of difference between the function. It is assumed that if number of samples is large, then the universal threshold may give better estimate for soft threshold [17].

\subsubsection{Visu Shrink}

Visu Shrink was introduced by Donoho [18]. It follows hard threshold rule. The drawback of this shrinkage is that neither speckle noise can be removed nor MSE can be minimized .It can only deal with additive noise [19]. Threshold T can be calculated using the formulae [20],

$$
T_{v}=\hat{\sigma} \sqrt{2 \log N}
$$




$$
\hat{\sigma}^{2}=\left[\frac{\operatorname{median}\left(\left|X_{i j}\right|\right)}{0.675}\right]^{2}, X_{i j} \in H H 1
$$

Where $\sigma$ is calculated as mean of absolute difference (MAD) which is a robust estimator and $\mathrm{N}$ represents the size of original image.

\subsubsection{Bayes Shrink}

The Bayes Shrink method has been attracting attention recently as an algorithm for setting different thresholds for every sub band. Here subbands refer to frequency bands that are different from each other in level and direction [21]. Bayes Shrink uses soft thresholding. The purpose of this method is to estimate a threshold value that minimizes the Bayesian risk assuming Generalized Gaussian Distribution (GGD) prior [12]. Bayes threshold is defined as [22],

$$
t_{B}=\sigma^{2} / \sigma_{s}
$$

Where $\sigma^{2}$ is the noise variance and $\sigma_{s}$ is signal variance without noise.

From the definition of additive noise we have,

$$
w(x, y)=s(x, y)+n(x, y)
$$

Since the noise and the signal are independent of each other, it can be stated that,

$$
\sigma_{w}^{2}=\sigma_{s}^{2}+\sigma^{2}
$$

$\sigma_{w}^{2}$ can be computed as shown below:

$$
\sigma_{w}^{2}=\frac{1}{n^{2}} \sum_{x, y=1}^{n} w^{2}(\mathrm{x}, \mathrm{y})
$$

The variance of the signal, $\sigma_{s}{ }^{2}$ is computed as

$$
\sigma_{s}=\sqrt{\max \left(\sigma_{w}^{2}-\sigma^{2}, 0\right)}
$$

\section{Simulation Results}

Simulated results have been carried on Cameraman image by adding two types of noise such as Gaussian noise and Speckle noise. The level of noise variance has also been varied after selecting the type of noise. Denoising is done using two filters Mean filter and Median filter and three Wavelet based methods i.e. Universal threshold, Visu shrink and Bayes shrink. Results are shown through comparison among them. Comparison is being made on basis of some evaluated parameters. The parameters are Peak Signal to noise Ratio (PSNR) and Mean Square Error (MSE).

$$
\begin{aligned}
& \text { PSNR }=10 \log _{10}\left(\frac{255^{2}}{M S E}\right) \mathrm{db} \\
& \text { MSE }=\frac{1}{M N} \sum_{i=1}^{M}(\mathrm{x}, \mathrm{y}) \sum_{j=1}^{N}(\mathrm{X}(\mathrm{i}, \mathrm{j})-P(i, j))^{2}
\end{aligned}
$$


Where, M-Width of Image, $\quad$ N-Height of Image

P- Noisy Image , $\quad$ X-Original Image

Table 1 and Table 2 show the comparison of PSNR and MSE for cameraman image at various noisevariancies. Figure6 and Figure 7 shows that bayes shrinkage has better PSNR and low MSE than filtering methods and other wavelet based thresholding techniques.

Table1. Comparison of PSNR for Cameraman image corrupted with Gaussian and Speckle noise at different Noise variances using db1 (Daubechies Wavelet)

\begin{tabular}{|c|c|c|c|c|c|c|}
\hline \multicolumn{7}{|c|}{ PSNR (PEAK Signal To Noise Ratio) } \\
\hline NOISE & $\begin{array}{c}\text { NOISE } \\
\text { VARIANCE }\end{array}$ & $\begin{array}{l}\text { MEAN } \\
\text { FILTER }\end{array}$ & $\begin{array}{c}\text { MEDIAN } \\
\text { FILTER }\end{array}$ & $\begin{array}{c}\text { UNIVERSAL } \\
\text { THRESHOLD }\end{array}$ & $\begin{array}{c}\text { VISU } \\
\text { SHRINK }\end{array}$ & $\begin{array}{c}\text { BAYES } \\
\text { SHRINK }\end{array}$ \\
\hline \multirow{6}{*}{ 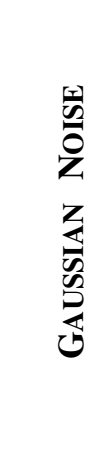 } & 0.001 & 24.0598 & 25.4934 & 27.2016 & 28.2978 & 33.7031 \\
\hline & 0.002 & 23.2251 & 24.3480 & 25.1748 & 26.1439 & 29.9001 \\
\hline & 0.003 & 22.5261 & 23.4147 & 24.0062 & 24.8430 & 27.7650 \\
\hline & 0.004 & 21.9796 & 22.6049 & 23.1590 & 23.8149 & 26.0865 \\
\hline & 0.005 & 21.4536 & 22.0205 & 22.5099 & 23.0527 & 25.1235 \\
\hline & 0.01 & 19.5569 & 19.7703 & 20.3580 & 20.5660 & 22.0446 \\
\hline \multirow{6}{*}{ 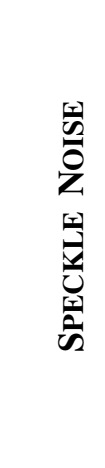 } & 0.001 & 24.8274 & 26.6157 & 28.4073 & 32.6526 & 44.0220 \\
\hline & 0.002 & 24.5114 & 26.1260 & 26.8834 & 30.4768 & 40.0535 \\
\hline & 0.003 & 24.2207 & 25.6708 & 25.9557 & 29.3585 & 38.3935 \\
\hline & 0.004 & 23.9316 & 25.2771 & 25.3274 & 28.1881 & 35.6827 \\
\hline & 0.005 & 23.7015 & 24.8599 & 24.8691 & 27.5283 & 34.3460 \\
\hline & 0.01 & 22.6357 & 23.4053 & 23.3231 & 25.1853 & 30.9207 \\
\hline
\end{tabular}




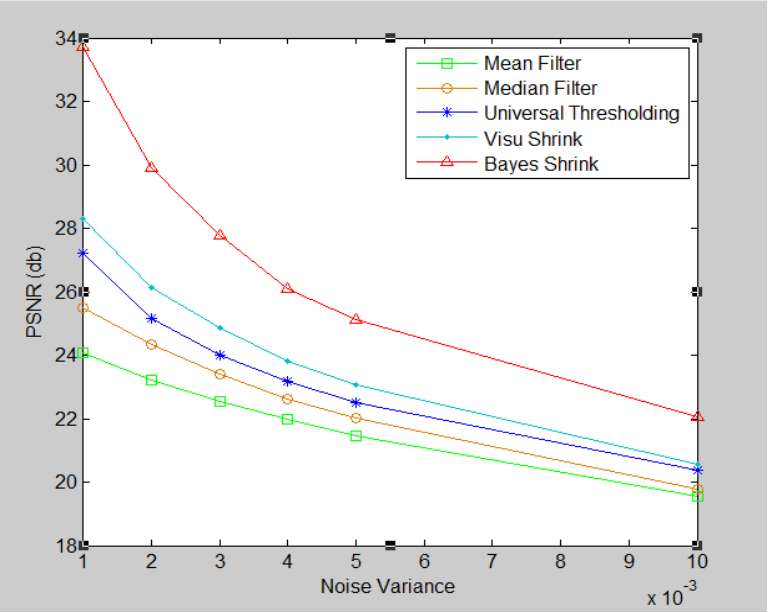

Figure6. Comparison of PSNR for cameraman image (corrupted with Gaussian noise) at different noise variance

Table2. Comparison of MSE for Cameraman image corrupted with Gaussian and Speckle noise at different Noise variances using db1

\begin{tabular}{|c|c|c|c|c|c|c|}
\hline \multicolumn{7}{|c|}{ MSE (MEAN SQUARE ERROR) } \\
\hline NOISE & $\begin{array}{c}\text { NOISE } \\
\text { VARIANCE }\end{array}$ & $\begin{array}{l}\text { MEAN } \\
\text { FILTER }\end{array}$ & $\begin{array}{l}\text { MEDIAN } \\
\text { FILTER }\end{array}$ & $\begin{array}{l}\text { UNIVERSAL } \\
\text { THRESHOLD }\end{array}$ & $\begin{array}{c}\text { VISU } \\
\text { SHRINK }\end{array}$ & $\begin{array}{c}\text { BAYES } \\
\text { SHRINK }\end{array}$ \\
\hline \multirow{6}{*}{ 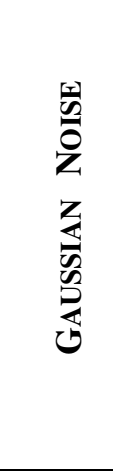 } & 0.001 & 255.3265 & 183.5446 & 123.8560 & 96.2288 & 27.7188 \\
\hline & 0.002 & 309.4321 & 238.9368 & 197.5136 & 158.0136 & 66.5377 \\
\hline & 0.003 & 363.4693 & 296.2178 & 258.5006 & 213.1975 & 108.7875 \\
\hline & 0.004 & 412.2133 & 356.9362 & 314.1828 & 270.1428 & 160.1160 \\
\hline & 0.005 & 465.2894 & 408.3482 & 364.8271 & 321.9641 & 199.8629 \\
\hline & 0.01 & 720.1005 & 685.5656 & 598.8007 & 570.7912 & 406.0842 \\
\hline \multirow{6}{*}{ 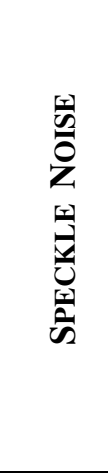 } & 0.001 & 213.9645 & 141.7451 & 93.8319 & 35.3036 & 2.5756 \\
\hline & 0.002 & 230.1138 & 158.6638 & 133.2721 & 58.2642 & 6.4229 \\
\hline & 0.003 & 246.0413 & 176.1971 & 165.0083 & 75.3748 & 9.4130 \\
\hline & 0.004 & 262.9796 & 192.9158 & 190.6971 & 98.6903 & 17.5716 \\
\hline & 0.005 & 277.2851 & 212.3693 & 211.9193 & 114.8823 & 23.9047 \\
\hline & 0.01 & 354.4109 & 296.8613 & 302.5347 & 197.0393 & 52.6035 \\
\hline
\end{tabular}




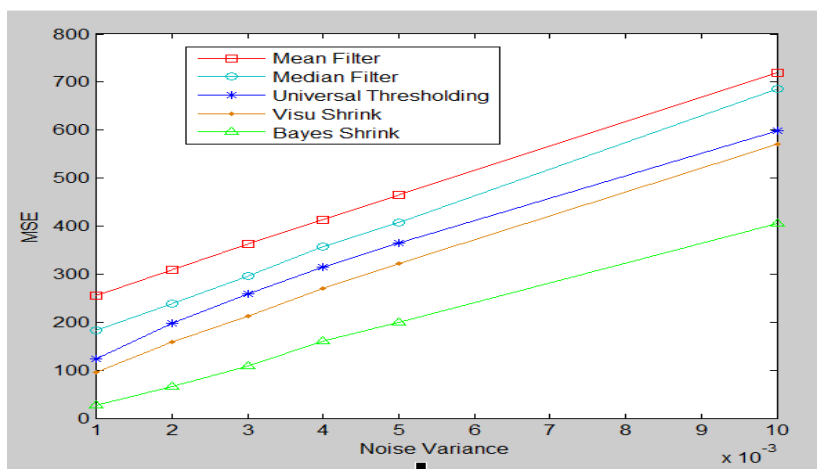

Figure 7. Comparison of MSE for cameraman image (corrupted with Gaussian noise) at different noise variances

The cameraman image is corrupted by gaussian noise of variance 0.01 and results obtained using filters and wavelets have been shown in Figure 8.

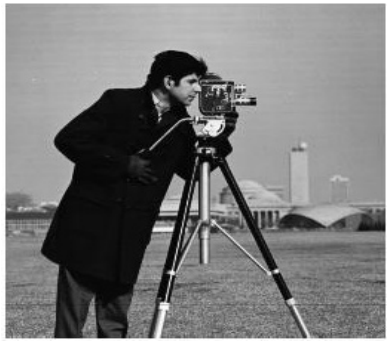

(a)

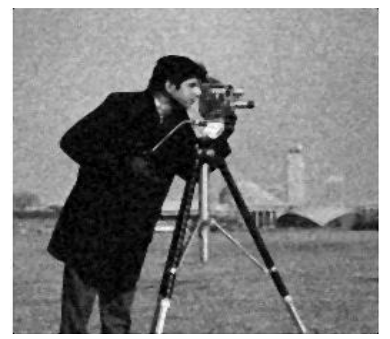

(d)

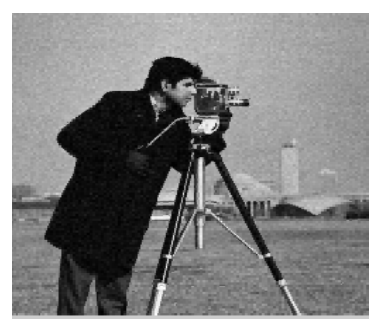

(g)

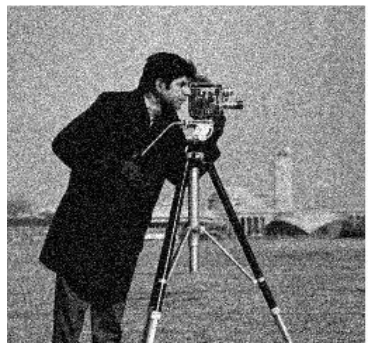

(b)

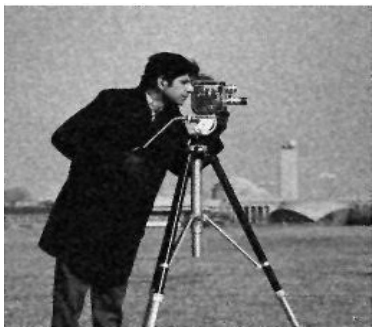

(e)

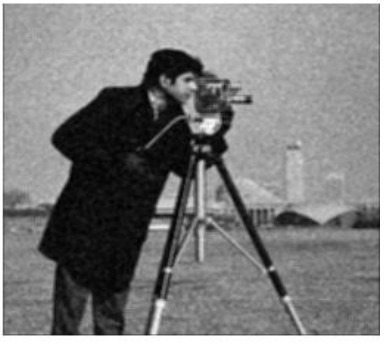

(c)

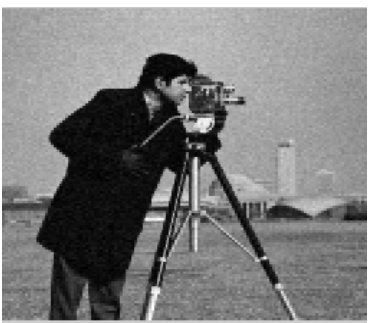

(f)

Figure 8. Denoising of cameraman image corrupted by Gaussian noise of variance 0.01 (a) Original image (b) Noisy image $\quad$ (c) Mean Filter (d) Median Filter (e) Universal Thresholding (f) Visu Shrink (g) Bayes shrink 
A Comparative study of various wavelet families viz. Daubechies, Symlet, Coiflet, Biorthogonal and Reverse Biorthogonal using the Matlab Wavelet Tool box function wfilters is done and results have been tabulated in Table 3. Almost all the wavelet families perform in a much similar fashion.

Table3. Comparison of MSE and PSNR for Cameraman image (with Gaussian noise of variance 0.001) using various Wavelet families namely Daubechies, Symlet, Coiflet, Biorthogonal and Reverse Biorthogonal.

\begin{tabular}{|c|c|c|c|c|c|c|c|}
\hline \multirow{2}{*}{\multicolumn{2}{|c|}{$\begin{array}{l}\text { WAVELET } \\
\text { FAMILIES }\end{array}$}} & \multicolumn{3}{|c|}{ MSE } & \multicolumn{3}{|c|}{ PSNR } \\
\hline & & \multirow{2}{*}{$\begin{array}{c}\begin{array}{r}\text { UNIVERSAL } \\
\text { THRESHOLD }\end{array} \\
118.9888\end{array}$} & \multirow{2}{*}{$\begin{array}{c}\text { VISU } \\
\text { SHRINK } \\
92.7006\end{array}$} & \multirow{2}{*}{$\begin{array}{l}\text { BAYES } \\
\text { SHRINK } \\
27.8870\end{array}$} & \multirow{2}{*}{$\begin{array}{c}\begin{array}{c}\text { UNIVERSAL } \\
\text { THRESHOLD }\end{array} \\
27.3757\end{array}$} & \multirow{2}{*}{$\begin{array}{c}\text { VISU } \\
\text { SHRINK } \\
28.4600\end{array}$} & \multirow{2}{*}{$\begin{array}{r}\text { BAYES } \\
\text { SHRINK } \\
33.6768\end{array}$} \\
\hline \multirow{5}{*}{ 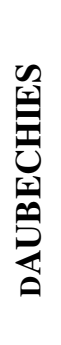 } & $\mathrm{db} 2$ & & & & & & \\
\hline & $\mathrm{db} 5$ & 116.0008 & 91.0493 & 29.1175 & 27.4862 & 28.5380 & 33.4893 \\
\hline & $\mathrm{db} 7$ & 114.5742 & 93.8306 & 32.3802 & 27.5399 & 28.4074 & 33.0280 \\
\hline & db9 & 117.1231 & 96.3611 & 33.6797 & 27.4444 & 28.2918 & 32.8571 \\
\hline & db10 & 117.7054 & 97.1057 & 33.8515 & 27.4228 & 28.2584 & 32.8350 \\
\hline \multirow{5}{*}{$\sum_{\substack{n \\
=}}^{\infty}$} & sym2 & 118.9952 & 93.2712 & 30.7511 & 27.3755 & 28.4333 & 33.2522 \\
\hline & sym4 & 114.9689 & 91.2290 & 29.3524 & 27.5250 & 28.5295 & 33.4544 \\
\hline & sym6 & 113.4957 & 92.9196 & 30.9472 & 27.5810 & 28.4497 & 33.2246 \\
\hline & sym7 & 112.3352 & 89.5128 & 29.1537 & 27.6256 & 28.6120 & 33.4839 \\
\hline & sym8 & 111.7177 & 90.6427 & 30.6893 & 27.6496 & 28.5575 & 33.2609 \\
\hline \multirow{5}{*}{ 衰 } & coif1 & 119.0472 & 93.1594 & 27.9323 & 27.3736 & 28.4385 & 33.6697 \\
\hline & coif2 & 113.9656 & 89.6841 & 29.1131 & 27.5631 & 28.6036 & 33.4899 \\
\hline & coif3 & 112.4675 & 92.3045 & 29.8983 & 27.6205 & 28.4786 & 33.3743 \\
\hline & coif4 & 112.3909 & 91.2025 & 31.0492 & 27.6235 & 28.5307 & 33.2103 \\
\hline & coif5 & 112.2086 & 90.1873 & 30.9109 & 27.6305 & 28.5794 & 33.2297 \\
\hline \multirow{5}{*}{ 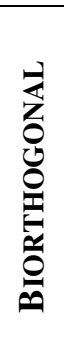 } & bior1.3 & 124.8644 & 99.1098 & 28.1472 & 27.1664 & 28.1696 & 33.6365 \\
\hline & bior2.2 & 125.0148 & 79.3262 & 22.2066 & 27.1612 & 29.1366 & 34.6660 \\
\hline & bior3.1 & 145.9058 & 85.0012 & 28.1984 & 26.4901 & 28.8366 & 33.6286 \\
\hline & bior4.4 & 114.5491 & 88.4300 & 29.0607 & 27.5409 & 28.6648 & 33.4977 \\
\hline & bior6.8 & 114.2567 & 88.5645 & 29.8665 & 27.5520 & 28.6582 & 33.3790 \\
\hline \multicolumn{2}{|c|}{ 空 } & 117.1884 & 98.8170 & 35.9098 & 27.4420 & 28.1825 & 32.5787 \\
\hline
\end{tabular}




\begin{tabular}{|l|l|l|l|l|l|l|l|}
\hline & rbio2.4 & 106.7042 & 109.6627 & 47.6843 & 27.8490 & 27.7302 & 31.3470 \\
\cline { 2 - 7 } & rbio3.3 & 104.6786 & 155.5353 & 75.9330 & 27.9322 & 26.2125 & 29.3265 \\
\cline { 2 - 7 } & & & & & & \\
\hline & rbio5.5 & 119.0634 & 82.0170 & 22.4013 & 27.3730 & 28.9918 & 34.6281 \\
\hline & & & & & & 33.1186 \\
\hline
\end{tabular}

\section{CONCLUSION}

In this paper, an analysis of denoising techniques like filters and wavelet methods has been carried out. Filtering is done by Mean and Median Filter. And three different wavelet thresholding techniques have been discussed i.e. Universal Thresholding, Bayes Shrink and Visu Shrink. From the simulation results, it is evident that Bayes shrinkage method has high PSNR at different noise variance and low MSE. This concludes that this method performs better in removing Gaussian noise and Speckle noise than filters and other wavelet methods.

\section{REFERENCES}

[1] Rajni, Anutam, "Image Denoising Techniques -An Overview," International Journal of Computer Applications (0975-8887), Vol. 86, No.16, January 2014.

[2] Akhilesh Bijalwan, Aditya Goyal and Nidhi Sethi, "Wavelet Transform Based Image Denoise Using Threshold Approaches," International Journal of Engineering and Advanced Technology (IJEAT), ISSN: 2249-8958, Vol.1, Issue 5, June 2012.

[3] S.Arivazhagan, S.Deivalakshmi, K.Kannan, "Performance Analysis of Image Denoising System for different levels of Wavelet decomposition," International Journal of Imaging Science and Engineering (IJISE), Vol.1, No.3, July 2007.

[4] Jappreet Kaur, Manpreet Kaur, Poonamdeep Kaur, Manpreet Kaur, "Comparative Analysis of Image Denoising Techniques," International Journal of Emerging Technology and Advanced Engineering, ISSN 2250-2459, Vol. 2, Issue 6, June 2012.

[5] Pawan Patidar, Manoj Gupta,Sumit Srivastava, Ashok Kumar Nagawat, "Image De-noising by Various Filters for Different Noise," International Journal of Computer Applications, Vol.9, No.4, November 2010.

[6] Govindaraj.V, Sengottaiyan.G , "Survey of Image Denoising using Different Filters," International Journal of Science, Engineering and Technology Research (IJSETR) ,Vol.2, Issue 2, February 2013.

[7] Idan Ram, Michael Elad, "Generalized Tree-Based Wavelet Transform," IEEE Transactions On Signal Processing, Vol. 59, No. 9, September 2011.

[8] Rakesh Kumar and B.S.Saini, "Improved Image Denoising Techniques Using Neighbouring Wavelet Coefficients of Optimal Wavelet with Adaptive Thresholding," International Journal of Computer Theory and Engineering, Vol.4, No.3, June 2012.

[9] Sethunadh R and Tessamma Thomas, "Spatially Adaptive image denoising using Undecimated Directionlet Transform," International Journal of Computer Applications, Vol.84, No. 11,December 2013

[10] S.Kother Mohideen Dr. S. Arumuga Perumal, Dr. M.Mohamed Sathik , “ Image De-noising using Discrete Wavelet transform," IJCSNS International Journal of Computer Science and Network Security, Vol.8 No.1, January 2008.

[11] Savita Gupta, R.C. Chauhan and Lakhwinder Kaur, "Image denoising using Wavelet Thresholding," ICVGIP 2002, Proceedings of the Third Indian Conference on Computer Vision, Graphics Image Processing, Ahmedabad, India, 2002

[12] S.Grace Chang, Bin Yu, Martin Vetterli , "Adaptive Wavelet Thresholding for image denoising and compression,” IEEE Transaction On Image Processing, Vol.9, No.9, September 2000 
[13] Nilanjan Dey, Pradipti Nandi, Nilanjana Barman, Debolina Das, Subhabrata Chakraborty ,“ A Comparative Study between Moravec and Harris Corner Detection of Noisy Images Using Adaptive Wavelet Thresholding Technique," International Journal of Engineering Research and Applications (IJERA), ISSN: 2248-9622, Vol. 2, Issue 1, Jan-Feb 2012.

[14] Tajinder Singh, Rajeev Bedi, "A Non - Linear Approach For Image De-Noising Using Different Wavelet Thresholding," International Journal of Advanced Engineering Research and Studies, ISSN2249-8974,Vol.1,Issue3,April-June,2012

[15] Abdolhossein Fathi and Ahmad Reza Naghsh-Nilchi, "Efficient Image Denoising Method Based on a New Adaptive Wavelet Packet Thresholding Function," IEEE Transaction On Image Processing, Vol. 21, No. 9, September 2012

[16] Virendra Kumar, Dr. Ajay Kumar, "Simulative Analysis of Image denoising using Wavelet ThresholdingTechnique," International Journal of Advanced Research in Computer Engineering and Technology (IJARCET), Vol.2, No.5, May 2013

[17] Mark J.T. Smith and Steven L. Eddins, "Analysis/SynthesisTechniques for subband image coding," IEEE Trans. Acoustic Speech and Signal Processing, Vol.38, No.8, Aug 1990

[18] D.L. Donoho and I.M. Johnstone, "Denoising by soft thresholding," IEEE Trans. on Information Theory, Vo.41, 1995

[19] Raghuveer M. Rao, A.S. Bopardikar Wavelet Transforms: Introduction to Theory and Application published by Addison-Wesley, 2001

[20] S.Sutha, E. Jebamalar Leavline, D. ASR Antony Gnana Singh, " A Comprehensive Study on Wavelet based Shrinkage Methods for Denoising Natural Images," WSEAS Transactions on Signal Processing, Vol. 9, Issue 4, October 2013

[21] E.Jebamalar Leavline, S.Sutha, D.Asir Antony Gnana Singh, "Wavelet Domain Shrinkage Methods for Noise Removal in Images: A Compendium," International Journal of Computer Applications,Vol.33, No.10, November 2011

[22] G.Y. Chen, T.D. Bui, A. Krzyak, "Image denoising using neighbouring Wavelet coefficients," Acoustics Speech and Signal processing, IEEE International Conference, Vol.2, May 2004

\section{AUTHORS}

Anutam

She is currently pursuing M.Tech from SBS State TechnicalCampus, Ferozepur, India. She has completed B.Tech from PTUJalandhar in 2012. Her areas of interest includes Wireless Communication and Image Processing.

Mrs. Rajni

She is currently Associate Professor at SBS StateTechnical Campus Ferozepur, India. She has completed her M.E. from NITTTR, Chandigarh, India and B.Tech from NIT,KurukshetraIndia. She has fourteen years of academic experience. She has authored a number of research papers in International journals, National and International conferences. Her areas of interes include Wireless communication and Antenna design.
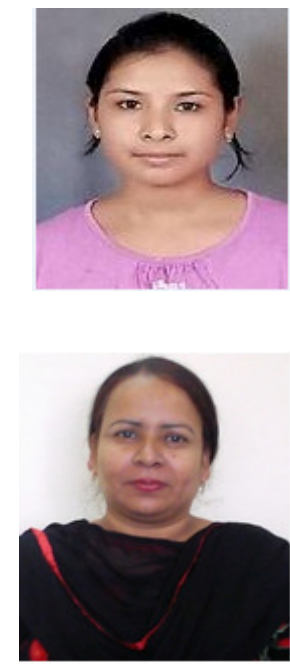